\title{
DESIGN TECHNIQUE FOR CALCULATING FUNCTIONAL PARAMETERS OF A ROTATING SHAPED CHARGE WITH PRELIMINARY HEATED LINER
}
A.S. Steshin
astboom@yandex.ru
A.V. Babkin
pc-os@bmstu.ru

Bauman Moscow State Technical University, Moscow, Russian Federation

\begin{tabular}{l} 
Abstract \\
The paper considers a separate issue of the shaped \\
charge functioning, i.e., functioning of a rotating charge elongation, rotation, centrifugal \\
under preliminary thermal action on its liner. Estimates destruction \\
of two oppositely directed factors are provided: 1) in- \\
crease in the shaped-charge jet dynamic plasticity (limit \\
elongation coefficient); 2 ) increase in the jet susceptibil- \\
ity to centrifugal destruction. These factors are activated \\
by preliminary thermal action on the shaped charge \\
liner in the jet heated in excess of usual parameters. The \\
so-called "thermal" increase in the limit elongation \\
coefficient was estimated using empirical and theoretical \\
dependences of this value on the shaped-charge jet \\
parameters, and on the characteristics of its material. \\
Strength dependence on temperature was accepted as \\
linearly decreasing. Centrifugal factor was estimated \\
based on the law of kinetic moment conservation taking \\
into consideration the gradient nature of stretching and, \\
up to a certain point, the radial thinning of jet elements. \\
The moment of centrifugal and strength forces relation- \\
ship reaching the critical value was accepted as the be- \\
ginning of the jet element centrifugal destruction. From \\
this time moment the jet radial extension started. The \\
law of decompaction of its enlarging part was taken \\
from studies previously conducted by the authors. It was \\
demonstrated that the two considered factors acting in \\
the opposite directions in a jet were in compliance with Received 06.03 .2019 \\
each other ensuring optimal preliminary heating of the Accepted 04.04 .2019 \\
liner and penetration effect with a local maximum \\
\hline
\end{tabular}

Introduction. Shaped charges (SC) improvement and issues concerning optimization thereof in order to achieve certain goals continue to arouse the interest of researchers specializing in the explosion cumulative effect. 
A.S. Steshin, A.V. Babkin

Development proceeds both in traditional areas (for example, search for new materials in cumulative liner [1-3] and taking into account the influence of imperfections in the cumulative assembly shape $[4,5]$ ), as well as in nontraditional approaches (thermal or electromagnetic effects on the shaped-charge liner (SCL) or on the shaped-charge jet (SCJ) created by a charge [6-10]). Most of these studies consider shaped charges under stationary conditions, and less commonly rotating shaped charges is examined. The listed factors with respect to SC could be considered as different control factors. But could they be interconnected with each other? For example, could it be possible to expect a positive effect under simultaneous thermal effect on SCL and rotation of the SC; as of today, this issue still remains unclear.

The idea of a shaped charge with preliminary thermal effect on its liner is known since the 1990s [6,7]. Effect on the liner was carried out using a gas generator with time advance in regard to the detonation, and in such a way not to cause thermal initiation of the explosive [8]. SCL preliminary heating affects the SCJ temperature $\Delta T$ increase. This leads to an increase in dynamic plasticity, which indicator is the coefficient of the jet limit elongation $n_{\text {lim }}[1]$. An increase in the length $n l_{0} \leq n_{\text {lim }} l_{0}$ of jet penetrating into the target leads to increasing the penetration depth $L$ into the target, which was registered in Ref. [6, 7]. The jet penetration depth $L$ into the target is influenced not only by the jet length, but also by other parameters, in particular, by the jet density $\rho_{j}$ and by the target density $\rho_{t}$ that are interconnected between each other according to the Lavrentiev's formula

$$
L=n l_{0} \sqrt{\frac{\rho_{\mathrm{j}}}{\rho_{\mathrm{t}}}} .
$$

However, the known experimental data $[6,7]$ on the increase in penetrating effect of a thermal SC were obtained under stationary conditions, i.e., an SC under experiment was immobile. At the same time, real SC as part of more complex devices is in motion and often rotates in flight. Moreover, for a number of common explosive devices, the characteristic rotation frequency is relatively low, i.e., up to $30 \mathrm{~s}^{-1}$ [11]. But, as the SCL collapses during the SCJ generation and its stretching, the moment of inertia is continuously decreasing and the rotation frequency is increasing. With significant unwinding, volumetric centrifugal destruction of a jet is possible leading to a decrease in its average density $\rho_{\mathrm{j}}$ and, according to Lavrentiev, to a decrease in the penetrating ability $L$. Centrifugal jet destruction would be manifested the stronger, the lower material strength is, i.e., the dynamic yield strength $Y$. 
Thus, two opposite factors would appear in the operation of a rotating thermal SC under an increase in the liner initial heating $\Delta T$. These are SCJ additional thermal elongation and its material thermal and centrifugal decompaction. As of today, it still remained unclear, which of these factors would prevail. It was necessary to give an answer to this question; and this was the work purpose.

Techniques for calculating parameters of the rotating shaped charge action. Our study was carried out by calculation and theoretical approach for calculating the effect of a SC with thin-walled liner, i.e., the V.M. Marinin approach [12]. In addition to traditional calculation of the major cumulation parameters [1, 12], thermal and rotational effects were calculated [13, 14]. Thus, the SCJ thermal elongation was determined based on the ratios for coefficients of current $(n)$ and limit $\left(n_{\text {lim }}\right)$ elongation:

$$
\begin{gathered}
n=\left\{\begin{array}{ccc}
1+\dot{\varepsilon}_{z 0} t & \text { at } & 1+\dot{\varepsilon}_{z 0} t<n_{\mathrm{lim}} ; \\
n_{\lim }=A+B R_{0} \dot{\varepsilon}_{z 0} & \text { at } & 1+\dot{\varepsilon}_{z 0} t \geq n_{\mathrm{lim}},
\end{array}\right. \\
n_{\lim }=5.36\left(\frac{\rho_{\mathrm{j}} \dot{\varepsilon}_{z 0}^{2} R_{0}^{2}}{Y}\right)^{0.39},
\end{gathered}
$$

where $t$ is the current time; $\dot{\varepsilon}_{z 0}$ is the initial axial velocity gradient; $R_{0}$ is the SCJ element initial radius; $Y$ is the material strength characteristic under the jet conditions (dynamic yield strength of the material); $A$ and $B$ are empirical constants.

Formulas (2) are the formulas for current and limit elongation coefficients that are not containing thermal characteristics. Theoretical formula (3) for the limit elongation contains a value that could be associated with the heating characteristics [1]. This is the dynamic yield strength $Y$; which nature of the temperature dependence is known

$$
Y=Y_{0}\left(1-\frac{T}{T_{\mathrm{m}}}\right)
$$

where $Y_{0}$ is the yield strength under normal conditions; $T$ is the current temperature; $T_{\mathrm{m}}$ is the melting temperature [15].

Relations (3) and (4) make it possible to establish the interrelationship between the values in natural conditions, when $\Delta T=0$ preliminary heating is missing, and when it is present

$$
Y_{\text {nat }}=Y_{0}\left(1-\frac{T_{\text {nat }}}{T_{\mathrm{m}}}\right), Y_{\mathrm{h}}=Y_{0}\left(1-\frac{T_{\mathrm{nat}}+\Delta T}{T_{\mathrm{m}}}\right)
$$




$$
\begin{gathered}
n_{\text {lim.h }}=n_{\text {lim.nat }}\left(\frac{Y_{\text {nat }}}{Y_{\mathrm{h}}}\right)^{0.39}=n_{\text {lim.nat }}\left(\frac{1-\frac{T_{\text {nat }}}{T_{\mathrm{m}}}}{1-\frac{T_{\text {nat }}+\Delta T}{T_{\mathrm{m}}}}\right)^{0.39}= \\
=n_{\text {lim.nat }}\left(\frac{T_{\mathrm{m}}-T_{\text {nat }}}{T_{\mathrm{m}}-T_{\text {nat }}-\Delta T}\right)^{0.39} \cdot
\end{gathered}
$$

The last expression and the experimental formula (2) are leading to a dependence of the limit elongation coefficient $n_{\text {lim }}$ not only on the initial gradient and radius, but also on the preliminary heating temperature:

$$
n_{\text {lim.h }}=\left(A+B \dot{\varepsilon}_{z 0} R_{0}\right)\left(\frac{T_{\mathrm{m}}-T_{\text {nat }}}{T_{\mathrm{m}}-T_{\text {nat }}-\Delta T}\right)^{0.39} .
$$

This dependence was particularly used to account for the effect of heating on the jet dynamic plasticity.

The dynamic yield strength $Y$, not only affects the jet limit elongation $n_{\text {lim }}$, but also determines the jet behavior during its rotation and its probable centrifugal destruction. Main calculated relationships describing a rotating SC are briefly as follows [14].

Prior to the start of centrifugal destruction, the jet radial thinning parameters during its axial elongation are assumed to being the same as those of a non-rotating jet, i.e., SCJ element current elongation coefficient $n=l / l_{0}=$ $=R_{0}^{2} / R^{2}=1+\dot{\varepsilon}_{z 0} t$. It was assumed that the kinetic moment was maintained for any particle $d m$ located at the $R$ distance from the axis of rotation: $K_{d m}=\omega R^{2} d m / 2$. The law of a stretching jet untwisting from the initial angular velocity $\omega_{0}$, was expressed by the following formula:

$$
\omega=\omega_{0} \frac{R_{0}^{2}}{R^{2}}=\omega_{0} \frac{l_{0} R_{0}^{2}}{l R^{2}} \frac{l}{l_{0}}=\omega_{0} n=\omega_{0}\left(1+\dot{\varepsilon}_{z 0} t\right) .
$$

The moment of beginning of the rotating jet centrifugal destruction was determined by fulfillment of the critical condition:

$$
\left(\frac{\rho \omega^{2} R^{2}}{Y_{0}}\right)_{\mathrm{cr}}=C_{\mathrm{cr}}
$$

which is reduced by estimation results according to the previously considered models of high-gradient rods [13], where $C_{\mathrm{cr}}$ is a certain critical value of the current ratio between centrifugal and strength forces. Corresponding critical 
values of other parameters depend on the initial ratio of these forces and constitute:

critical radius

$$
R_{\mathrm{cr}}=R_{0} \sqrt{\frac{\rho \omega_{0}^{2} R_{0}^{2}}{Y_{0}} \frac{1}{C_{\mathrm{cr}}}} ;
$$

critical angular untwisting speed

$$
\omega_{\mathrm{cr}}=\frac{C_{\mathrm{cr}} Y_{0}}{\rho \omega_{0} R_{0}^{2}}
$$

critical elongation coefficient

$$
n_{\mathrm{cr}}=\frac{C_{\mathrm{cr}} Y_{0}}{\rho \omega_{0}^{2} R_{0}^{2}} .
$$

The ratio of the jet element initial radius $R_{0}$, and of the critical radius $R_{\mathrm{cr}}$, determines one or another element mode of operation, when it is elongated and untwisted. Algorithm for the corresponding calculation was presented in detail in works $[13,14]$. Let us restrict here to a simple example, where the radius $R_{0}$ of the generated jet element is in the following relationship with the $R_{\mathrm{cr}}$ critical radius characteristic of the given element with its initial angular velocity $\omega_{0}$ and material characteristics according to formula (5): $R_{0} \leq R_{\mathrm{cr}}$. From (5), it consequently follows:

$$
\frac{R_{\mathrm{cr}}}{R_{0}} \geq 1, \quad \sqrt{\frac{\rho \omega_{0}^{2} R_{0}^{2}}{Y_{0}} \frac{1}{C_{\mathrm{cr}}}} \geq 1, \quad \frac{\rho \omega_{0}^{2} R_{0}^{2}}{Y_{0}} \geq C_{\mathrm{cr}},
$$

i.e., the $R_{0} \leq R_{\mathrm{cr}}$ condition corresponds to such a situation, when at the moment of the jet element generation the initial ratio of centrifugal and strength forces exceedthe critical value. Physically, this is the beginning of the element centrifugal destruction from the moment of its generation. At $R_{0} \geq R_{\mathrm{cr}}$, initial ratio of centrifugal and strength forces is not sufficient for this, i.e., the jet should stretch, and the ratio $\rho \omega^{2} R^{2} / Y_{0}$ should raise up to the value $C_{\mathrm{cr}}$. In this case, jet centrifugal destruction could occur along the course of its elongation due to a decrease in the moment of inertia and the corresponding untwisting.

Algorithm for determining the disruptive action against a target by the SCJ elements, both partially destroyed and partially monolithic, is presented in Ref. $[13,14]$. Lavrentiev's formula (1) was taken as the basis, and it was further modified 


$$
L=l_{0}\left[k_{l}+\left(1-k_{l}\right) k_{v} \sqrt{\frac{\rho_{\mathrm{av}}}{\rho_{\mathrm{j} 0}}}\right] \sqrt{\frac{\rho_{\mathrm{j} 0}}{\rho_{\mathrm{t}}}},
$$

where $l_{0}$ is the jet element initial length; $k_{l}$ is the value characterizing part of the element length remaining and acting along the target; $1-k_{l}$ is the coefficient of the jet efficient length reduction due to its centrifugal destruction at the uniform stretching stage; $\rho_{\mathrm{av}}$ is the average density of the centrifugally destructed jet element; $\rho_{j 0}$ is the SCJ material normal density; $\rho_{t}$ is the target density.

The law of decompaction and alteration in time of the average density $\rho_{\mathrm{av}}$ of the jet centrifugally destroyed element has the form of the ratio between the following values:

$$
\frac{\rho_{\mathrm{av}}}{\rho_{\mathrm{j} 0}}=\frac{n_{\mathrm{cr}}}{n} \frac{R_{\mathrm{cr}}^{2}}{R^{2}}=\frac{n_{\mathrm{cr}}}{n} \frac{1}{1+0.2 \omega_{\mathrm{cr}}^{2}(\Delta t)^{2}}=\frac{n_{\mathrm{cr}}}{n} \frac{1}{1+0.2 \frac{\omega_{0}^{2} n_{\mathrm{cr}}^{2}}{\dot{\varepsilon}_{z 0}^{2}}\left(n-n_{\mathrm{cr}}\right)^{2}} .
$$

In formula (6) $k_{v}$ is the coefficient accounting for the effect of increasing the limit penetration velocity $v_{\text {lim }}$ of a target by a decompaction jet. When the jet velocity is higher than the limit velocity at the given density, the coefficient $k_{v}=1$, in the contrary case $k_{v}=0$. As far as dependence of the limit penetration velocity $v_{\text {lim }}$ on density is concerned, it is determined from the $\rho_{\mathrm{av}} v_{\mathrm{lim}}^{2}=\rho_{\mathrm{j} 0} v_{\mathrm{lim} 0}^{2}$ pressures equality condition is exerted on a target by monolithic and decompaction jets and according to the target limit penetration velocity by a monolithic jet [1] $\left(v_{\lim 0} \approx 2 \cdot 10^{3} \mathrm{~m} / \mathrm{s}\right.$ for a copper jet and a highstrength steel target).

The dynamic yield stress $Y$, which is main strength characteristic require for calculating the rotational effects, was determined on the basis of experimental data using the so-called critical mass velocity, i.e., the difference in axial velocity between the adjacent separate jet elements [16]:

$$
v_{\mathrm{cr}}=0.65 \sqrt{\frac{Y_{\text {nat }}}{\rho_{\mathrm{j} 0}}} .
$$

The specific values $v_{\mathrm{cr}}$ were taken from the known experimental data $[12,17]$, according to which, copper of varying quality (M1 copper, M0 oxygen-free copper) could possess the following critical velocity values: 90,110 and $130 \mathrm{~m} / \mathrm{s}$.

Results of thermal and centrifugal effects calculation. Results were obtained for a SC with a diameter of $72 \mathrm{~mm}$ [11]. Maximum possible rotation 
velocity was $30 \mathrm{~s}^{-1}$. Natural temperature of the copper SC was $500{ }^{\circ} \mathrm{C}$ (average over the generally accepted range of values is $400-600{ }^{\circ} \mathrm{C}$ [1]). Figure demonstrates the effect of preliminary heating $\Delta T$ of the liner on the $\bar{L}=L / L_{0}$ jet penetrating ability for three values of the critical mass velocity $v_{\mathrm{cr}}$ (or dynamic yield strength $Y$ ). Curve 1 corresponds to the nonrotating charge, curves 2-4 correspond to the rotating charge with various $v_{\mathrm{cr}}$ and $L_{0}$ (penetration capacity of the nonrotating and unheated charge) values.

Optimum heating for maximum penetration exists for all the values $v_{\mathrm{cr}}$. However, its contrast range increases with the growing jet material dynamic strength. Thus, for dynamic strength corresponding to the critical velocity difference of $90 \mathrm{~m} / \mathrm{s}$ (curve 2 in the Figure), the optimal heating is $260{ }^{\circ} \mathrm{C}$, and the SC penetration ability raises by about $10 \%$ compared to the unheated SC. Such heating could quite remain a long time [1], and without any use of the explosive thermal insulator (phlegmatized octogen [7]).

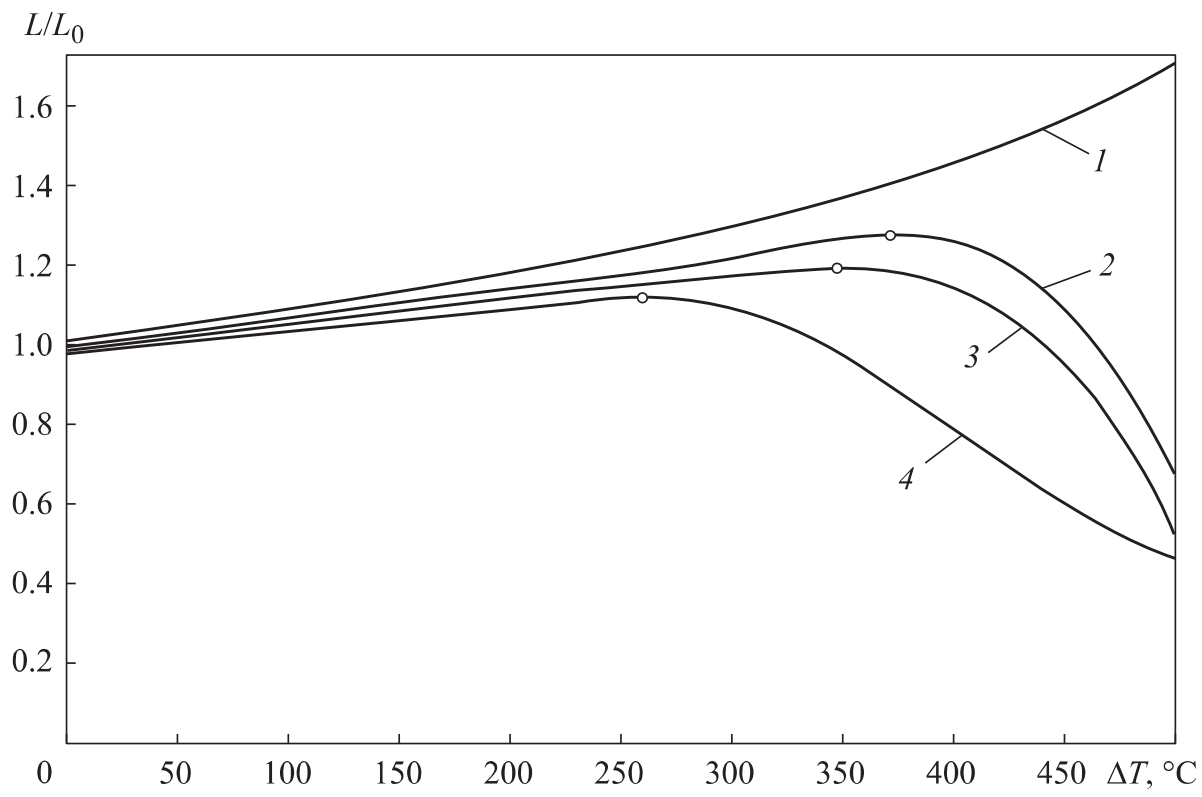

Penetration ability dependence of nonrotating and rotating SC depending on SCL preliminary heating at various indicators of the jet material dynamic strength:

$$
1 \text { at } \omega=0 ; 2-4 \text { at } \omega=30 \mathrm{~s}^{-1} \text { (at } v_{\mathrm{cr}}=90(2), 110(3) \text { and } 130(4) \mathrm{m} / \mathrm{s} \text { ) }
$$

As the dynamic strength increases $\left(v_{\mathrm{cr}}=110 \mathrm{~m} / \mathrm{s}\right)$, the optimal initial heating continues, but shifts toward higher values $\left(350^{\circ} \mathrm{C}\right)$, and the maximum expected penetration effect reaches $20 \%$ (curve 3 in the Figure). The observed extremum is not acute, and it appears at a slightly lower initial heating $\left(\approx 330^{\circ} \mathrm{C}\right)$, penetration capacity remains almost the same. In order to ensure 
A.S. Steshin, A.V. Babkin

such heating of the liner, a pulse action with a short admissible time period $(\sim 0.15 \mathrm{~s})$ is required [8]. It is necessary to synchronize enabling thermal effects on the SCL; and such initial heating of the charge liner with octogen munition could still be carried out without additional thermal insulation of the explosive. Even greater values of dynamic strength $\left(v_{\mathrm{cr}}=130 \mathrm{~m} / \mathrm{s}\right)$ continue the indicated tendency (curve 4 in the Figure); when deriving the heating time period values from the optimal values region, it would be necessary to introduce thermal insulation of the SC explosive [8].

It should be mentioned that similar penetration capacity of nonrotating SC exposed to preliminary thermal effect on the liner was achieved earlier [6, 7].

On the whole, a positive answer could be given to the question of a possibility in principle to create a rotating SC with the liner exposed to preliminary thermal effect under conditions of the oppositely directed factors action. Factors of the SCJ temperature-strain additional elongation and its temperature-centrifugal destruction and decompaction turned out to be comparable in significance over the jet life time. One of them predominates at certain moments ensuring the SCL optimal initial heating and the SC penetration capacity with a local maximum.

Conclusion. When the SC initial rotation is missing, calculation predicts that the preliminary heating of the charge liner could significantly increase its penetration capacity.

Initial rotation frequency of $30 \mathrm{~s}^{-1}$ significantly reduces the penetration capacity of a charge with preliminary heated liner. According to any estimation of the jet material dynamic strength $(Y=0.17-0.36 \mathrm{GPa}$ corresponds to $v_{\mathrm{cr}}=90-130 \mathrm{~m} / \mathrm{s}$ ), there appears optimal preliminary heating that provides the maximum penetration capacity. This maximum penetration is by $10-12 \%$ lower than penetration by a heated and nonrotating charge, but is by $15-28 \%$ higher than penetration by an unheated and nonrotating charge. The rotation factor devalues the thermal factor effect, but does not completely overcome it.

When assessing dynamic strength within the specified range of values, optimal preliminary heating of the liner lies in the range of $260-375^{\circ} \mathrm{C}$. At the lower value of $260^{\circ} \mathrm{C}$, the octogen charge could remain in this state for a long time period. At the upper value of $375^{\circ} \mathrm{C}$, thermal effect on the charge should be only short-term, and the octogen charge should be thermally insulated.

\section{REFERENCES}

[1] Orlenko L.P., ed. Fizika vzryva. T. 2 [Explosion physics. Vol. 2]. Moscow, Fizmatlit Publ., 2002. 
Design Technique for Calculating Functional Parameters of a Rotating Shaped Charge...

[2] Babkin A.V., Ladov S.V., Fedorov S.V. Behavior features of cumulative jets of composite powder liner in free flight. Oboronnaya tekhnika, 2007, no. 3-4, pp. 38-53 (in Russ.).

[3] Babkin A.V., Ladov S.V., Fedorov S.V. Analysis of influence of the sintered liner composition on the shaped-charge jet free flight behavior and its penetration capacity. Proc. 25th Int. Symp. Ballistics, 2010, vol. 2, pp. 959-966.

[4] Baskakov V.D., Zarubina O.V., Karnaukhov K.A., et al. Mathematical modelling of ideal flat jets impact. Herald of the Bauman Moscow State Technical University, Series Natural Sciences, 2016, no. 2 (65), pp. 79-90 (in Russ.).

DOI: https://doi.org/10.18698/1812-3368-2016-2-79-90

[5] Karnaukhov K.A., Baskakov V.D., Korenkov V.V., et al. Peculiarity of the shapedcharge liner collapse concerning the unevenness in its cross-section. J. Phys.: Conf. Ser., 2017, vol. 894, no. 1, art. 012039.

DOI: https://doi.org/10.1088/1742-6596/894/1/012039

[6] Kolpakov V.I., Pletnev S.L. [The effect of liner pre-heating on efficiency of shaped charge]. Tez. dokl. IV mezhdunar. konf. Lavrent'yevskie chteniya po matematike, mekhanike i fizike [Abs. IV Int. Conf. Lavrentiev Readings on Mathematics, Mechanics and Physics]. Novosibirsk, IG SO RAS Publ., 1995, pp. 125 (in Russ.).

[7] Korenkov V.V., Obukhov A.S., Smelikov V.G. Increase of steel target penetration by charge with preliminary heated liner. Dvoynye tekhnologii, 1999, no. 4, pp. 53-54 (in Russ.).

[8] Babkin A.V., Ladov S.V., Fedorov S.V., et al. Maximum allowable parameters of pulsed thermal impact on ring systems with energetic material. Oboronnaya tekhnika, 2000, no. 1-2, pp. 35-40 (in Russ.).

[9] Babkin A.V., Bondarenko P.A., Fedorov S.V., et al. [Limitations of the possibilities for increasing penetration of shaped charge at pulsed heating its liner]. Ekstremal'nye sostoyaniya veshchestva. Detonatsiya. Udarnye volny. Tr. mezhdunar. konf. III Kharitonovskie tematich. nauch. chteniya [Extreme States of the Substance. Detonation. Shock Waves. Proc. Int. Conf. III Khariton Theme Readings]. Sarov, VNIIEF Publ., 2002, pp. 257-263 (in Russ.).

[10] Matrosov A.D., Babkin A.V., Ladov S.V., et al. Influence of magnetic fields on shaped charge performance. Trans. PPPS 2001, 2001, pp. 182-186.

DOI: https://doi.org/10.1109/PPPS.2001.1002023

[11] Selivanov V.V., ed. Boepripasy. T. 1 [Ammunition. Vol. 1]. Moscow, BMSTU Publ., 2016.

[12] Marinin V.M., Babkin A.V., Kolpakov V.I. Calculation technique of cumulative charge performance parameters. Oboronnaya tekhnika, 1995, no. 4, pp. 34-39 (in Russ.).

[13] Babkin A.V., Rassokha S.S., Ladov S.V. On centrifugal destruction of gradient rods. Herald of the Bauman Moscow State Technical University, Series Mechanical Engineering, 2010, no. S, pp. 182-195 (in Russ.).

ISSN 1812-3368. Вестник МГТУ им. Н.Э. Баумана. Сер. Естественные науки. 2020. № 1 
A.S. Steshin, A.V. Babkin

[14] Babkin A.V., Rassokha S.S., Ladov S.V. Calculation method for functionality of the rotating shaped charges. Oboronnaya tekhnika, 2010, no. 1-2, pp. 23-30 (in Russ.).

[15] Johnson G.R., Cook W.H. A constitutive model and data for metals subjected to large strains, high strain rates and high temperatures. Proc. 7th Intern. Symp. Ballistics. Hague, Netherlands, 1983.

[16] Babkin A.V., Ladov S.V., Marinin V.M., et al. Regularities of the stretching and plastic failure of metal shaped-charge jets. J. Appl. Mech. Tech. Phys., 1999, vol. 40, iss. 4, pp. 571-580. DOI: https://doi.org/10.1007/BF02468430

[17] Svirskiy O.V., Vlasova M.A. Rating assessment of metals on the prospects of their use in cumulative charges liners. Izvestiya RARAN, 2006, no. 3 (48), pp. 83-88 (in Russ.).

Steshin A.S. - Student, Department of High Precision Flying Devices, Bauman Moscow State Technical University (2-ya Baumanskaya ul. 5, str. 1, Moscow, 105005 Russian Federation).

Babkin A.V. - Dr. Sc. (Eng.), Professor, Department of High Precision Flying Devices, Bauman Moscow State Technical University (2-ya Baumanskaya ul. 5, str. 1, Moscow, 105005 Russian Federation).

\section{Please cite this article as:}

Steshin A.S., Babkin A.V. Design technique for calculating functional parameters of a rotating shaped charge with preliminary heated liner. Herald of the Bauman Moscow State Technical University, Series Natural Sciences, 2020, no. 1 (88), pp. 61-70. DOI: https://doi.org/10.18698/1812-3368-2020-1-61-70 